\title{
Electro-Convulsive Therapy (ECT): an Egyptian perspective
}

\section{TA Okasha}

Institute of Psychiatry, Faculty of Medicine, Ain Shams University, World Psychiatric Association, Zonal Representative for Northern Africa, Cairo, Egypt

\begin{abstract}
The world celebrated 68 years of electroconvulsive therapy (ECT) recently. Whilst ECT has stood the test of time for nearly six decades. stigma remains one of the main issues that needs to be addressed. ECT is a technically advanced and effective treatment that is often misunderstood and maligned not only by the lay public but by psychiatrists as well. One of the main causes of stigma is the nomenclature which needs to be changed since shock and convulsion are absent when giving modified ECT.Based on the evidence derived from randomized controlled trials and extensive clinical experience for nearly 70 years, ECT is presently the most effective treatment for certain psychiatric disorders. The most transient memory disturbance is a moderate price for what can be achieved, as the benefit to risk ratio of ECT is usually favorable.The economic aspects of ECT use in Egypt, as well as the problem of ECT training, are discussed in this paper.The ethical aspects of ECT are reviewed as well as the discrepancies between developed and developing countries in its application, which needs to be addressed from a transcultural perspective.
\end{abstract}

Key Words: Electroconvulsive Therapy, Developing Countries, Stigma, Transcultural aspects

Received: 11.11 .2005

Accepted: 27.10.2006

\section{Introduction}

As the world celebrates 68 years of electroconvulsive therapy (ECT), there has been no other line of treatment in medicine that has stood the test of time for over six decades like ECT. The controversy over ECT is what enabled it to prove itself and helped it in its development to our present day as William Hazlitt said: "when a thing ceases to be a subject of controversy, it ceases to be a subject of interest".

Over the past years several teachers in psychiatry from different areas of the world both developed and developing have noticed that the training of junior psychiatrists in some parts of the world lacks training in ECT. Unfortunately, some resident training programs do not include ECT, which results in psychiatrists who have only read about ECT in textbooks and are, of course, reluctant to use it. Despite evidence of its efficacy and safety, it has been widely under-utilized, to the disadvantage of patients, their families, and to the health care budgets. In response, the World Psychiatric Association (WPA) has created a WPA Institutional Program on the Core Training

\section{Correspondence:}

Dr. Tarek A. Okasha

3, Shawarby Street, Kasr El Nil, Cairo, Egypt.

email : tokasha@internetegypt.com
Curriculum for Psychiatry, which states the minimum requirements for the education and training of a post graduate to become a specialist in psychiatry. ${ }^{1}$ This program has a special emphasis on training in the use of ECT with an emphasis on safety. Similarly, the WPA section on biological psychiatry has also prepared a Position Statement on the Use and Safety of ECT. The scope of this statement is to consider the evidence for the efficacy and safety of ECT in the treatment of psychiatric disorders as well as determining its optimum use with the continuation of pharmacotherapy and recommendations for good ECT practice. ${ }^{2}$

\section{ECT in Depression and Mania}

The use of ECT in depression is well established. 30 years ago, professors teaching psychiatry in Egypt emphasized the importance of using ECT as a first line of treatment in mania. Whilst at that time most specialists were not convinced of such application of the treatment, we now find guidelines of the American Psychiatric Association discussing the importance of use of ECT in patients suffering from mania stating that ECT in the treatment of mania is as effective as ECT in the treatment of depression. ${ }^{3}$ In an Egyptian study by Okasha and Ramy focusing on the economic aspects of ECT use in mania, 60 patients in both a university hospital and a private psychiatric hospital were studied. It was found that patients 
receiving ECT for the treatment of mania compared to patients not receiving ECT had a shorter hospital stay at 19 versus 38 days and that the cost of hospitalization was 850 USD compared to 1485 USD in the non ECT group. ${ }^{4}$ Both cost of treatment as well as duration of stay in hospital are critical factors in psychiatric health care. This is especially so in developing countries where the turn over in hospitals is high in order to accommodate patients in need of hospitalization whilst at the same time reducing hospitalization costs, which is essential due to budget constraints. Specifically given that the majority of patients in developing countries pay for these services themselves.

On the other hand developed countries have problems with third party payers regarding the duration of stay in hospital and cost of treatment whether for biological or psychological therapies.

\section{ECT and Stigma}

The stigma associated with ECT is a specific area that needs to be addressed given that ECT is both a technically advanced and effective treatment that is often misunderstood and maligned by not only the lay public but by psychiatrists as well. Between 1938 and the 1950's, there was extensive use of ECT. During that period of time, ECT was the major treatment, if not frequently the only biological treatment for mental illness. ${ }^{5}$ From the 1950's through the 1970's, with the advent of psychotropic medications (including the development of neuroleptics and of tricyclic antidepressants), a decline in the use of ECT was seen. In the 1970's, concerns emerged related to the side effects of psychotropic medications, including the cardiovascular effects of the tricyclic antidepressants and the potential for tardive dyskinesia with neuroleptics. This concern resulted in a resurgence of interest in ECT and led to many studies and reports evaluating the effectiveness of this modality.

At the same time, the myths, misinformation, and public outcry continued. Senator Eagleton lost his vice presidential bid when he revealed that he had received ECT. In the 1975 film "One Flew Over the Cuckoo's Nest", Jack Nicholson portrayed a patient receiving ECT for the wrong purpose (coercion) and in the wrong fashion (without anesthesia or muscle relaxant). While public concern continues as a result of negative media portrayal, progress in ECT has continued with significant medical advancements. ${ }^{6}$ Currently, there appears to a renewed interest in both ECT research and publications (not least of all since the emergence of the "Journal of ECT" in 1998) which has contributed to ECT entering the biological age of psychiatry.

\section{Modern ECT}

The issue of semantics is paramount. Modern ECT administered under anesthesia induces far less discomfort and medical sequelae than most surgical or pharmacological interventions. It is actually quite inappropriate to call such treatment "Shock therapy". Fink has pointed out that "Shock" has a specific meaning: it is the perception of the passage of an electric current. This produces pain and discomfort.

The word "Shock" denotes perceptions that do not occur under anesthesia. This would be analogous to labeling surgery "pain therapy". Little wonder such a term as "shock therapy" engenders stigma. ${ }^{7}$ Such outdated use of language leads to negative attitudes and prejudice. The words "convulsion" and "seizure" both have special meanings to the public. In studies of perceived social distance from different medical disorders, it was found that "convulsions" and "epilepsy" rank the same with "schizophrenia" in terms of negative attitude. ${ }^{8}$ Convulsions, as portrayed by the mass media, do not occur with modern ECT methods. "Seizure" is used in its' technical sense to refer to the patterned electrical response produced by an electrical stimulus.

Lack of awareness of the natural history of disorders treated by ECT in the Egyptian population make nearly $70 \%$ of families of patients believe that ECT is addictive and that once they receive ECT they will continue to relapse and never get better unless they receive another course of ECT (A Okasha, personal communication).

\section{The Future}

The treatment (ECT) should be given a new name that describes what is done in neutral, "unloaded" language. Words like "shock", "seizure" and "convulsive" should be eliminated. Several proposed terms such as "cerebroversion", analogous with "cardioversion", or "Central Stimulation" or "Central Stimulation with Patterned Response" (CSPR), or "Brain Synchronization Therapy" (BST), or "Brain Stimulation Therapy", or "Rhythm Restoration Therapy" might be effective in altering negative perceptions of ECT arising from semantic. . $^{9,10}$

In Egypt we have noticed, anecdotally, a change in refusal and stigma with a decrease in either after the introduction of these new names in the university hospitals. In addition families were more accepting of this treatment for a current episode of illness having on earlier admissions accepted the patient's admission to hospital only with the proviso that they not receive "electric treatment". Such observations do require formal study.

A clean break with the past appearsis in order. Specifically paying attention to semantic issues and the psychosocial origins of prejudice which may make this procedure more acceptable and thus available to many patients who need it.

Trainers and teachers in psychiatry worldwide should train all junior psychiatrists on the proper administration and use of ECT (or BST, as proposed), which can be a life saving intervention in many cases in psychiatry.

\section{Madrid Declaration}

According to the World Psychiatric Association's ethical guidelines known as the "Madrid Declaration", any psychiatrist who is not abreast of knowledge and withholds treatment from a patient is unethical. ${ }^{11}$

A "Principles" approach to biomedical ethics, describes and endorsed four such principles without a specific hierarchy. The principles are beneficence (doing good), nonmaleficence (not doing harm), autonomy (respect for the individual) and justice (being fair). ${ }^{12}$ Based on the evidence derived from randomized controlled trials and extensive clinical experience for nearly 70 years, ECT is, presently, the most effective treatment for certain psychiatric disorders. In consideration of what can be achieved, the most transient memory disturbance is a moderate price. The benefit to risk ratio of ECT is usually favorable. Respect of patient autonomy is the maxim of ECT.

Ottosson and Fink state that in most cases the use of ECT is 
in agreement with the principles of beneficence, nonmaleficence, and respect for autonomy. Sadly, the principle of justice is far from satisfied. ${ }^{13}$

\section{Conclusion}

While it might be anticipated that ECT will be replaced in the future by an effective and less controversial and prejudicial treatment, today it is an effective and safe treatment. All psychiatrists should be acquainted with ECT and be able to present the treatment to the patient and the patient's relatives in a knowledgeable and scientific manner and not simply as a last resort treatment after all other lines of treatment fail (as stated in some treatment algorhythms). An urgent goal of mental health care should be to provide access to ECT and eliminate the severe impediments to its use, so long as it can defend evidence based superiority over other treatments.

\section{References}

1. World Psychiatric Association: Institutional Program on the Core Curriculum for Psychiatry. WPA Yokohama, Japan (2002).

2. World Psychiatric Association: WPA Position Statement on the Use and Safety of ECT. WPA Cairo, Egypt (2005).

3. American Psychiatric Association The practice of electroconvulsive therapy: Recommendations for treatment, training, and privileging (2nd ed.) 2001

4. Okasha, T, Ramy H. Using Electro-convulsive Therapy (ECT) in the Treatment of Mania: Economical Aspects (read at the WPA economics meeting held in Cairo) (2001).

5. Fink PJ, Tasman A. Stigma and Mental illness. American Psychiatric Press. Inc. (1992)

6. Hay D.P. The stigma of Electroconvulsive Therapy: A workshop: Introduction. In Stigma and Mental Illness. Fink PJ, Tasman A. (Eds.) American Psychiatric Press Inc. (1992).

7. Fink M. Convulsive Therapy: Theory and Practice. Raven Press (1979).

8. Spiro, H. The Stigma of Electroconvulsive Therapy: Psychological and Semantic Aspects. In Stigma and Mental Illness. Fink PJ, Tasman A. (Eds.) American Psychiatric Press Inc. (1992).

9. Rakhawy YT. Electroconvulsive Therapy: A Synchronizing Remedy. Egypt. J. Psychiatry 1982;, 5: 17-21.

10. Okasha A. Okasha's Clinical Psychiatry. Anglo Egyptian Bookshop, Cairo (1988).

11. Okasha A, Arboleda-Florez J, Sartorius, N. Ethics Culture and Psychiatry International Perspectives. American Psychiatric Press (2000).

12. Beauchamp TL, Childress JF. Principles of biomedical ethics (5th ed). Oxford: Oxford University Press (2001).

13. Ottosson J, Fink M. Ethics in Electroconvulsive Therapy. BrunnerRoutledge. Taylor \& Francis Group (2004) 